\title{
New Physics Searches in the Top Quark Sector in Hadron Colliders
}

\author{
Pallabi Das* \\ on behalf of the ATLAS, CDF and CMS collaborations \\ Tata Institute of Fundamental Research, Mumbai \\ E-mail: pallabi.dasecern.ch
}

Several theories beyond the Standard Model of Particle Physics predict new and heavy particles which are yet to be observed in the hadron colliders. These particles are likely to decay through the top quark, making it suitable as a probe for new physics phenomena. In this report various experimental search strategies for such processes are discussed briefly. Important results from Tevatron, CMS and ATLAS experiments from the LHC are presented.

9th International Workshop on the CKM Unitarity Triangle

28 November - 3 December 2016

Tata Institute for Fundamental Research (TIFR), Mumbai, India

${ }^{*}$ Speaker. 


\section{Introduction}

The top quark is the heaviest known fundamental particle till date. With a mass of $173.34 \pm$ 0.27 (stat.) \pm 0.71 (syst.) $\mathrm{GeV}$ [1], it decays before it can hadronize, unlike other quarks. Due to its heavy mass the Yukawa coupling of top quark with Higgs is expected to be large. The top quark may also serve as the decay product of other heavier particles predicted in theories beyond SM which are not yet discovered. In this way top quark can act like a probe for new physics phenomena, making it an interesting particle to study.

In the following sections, searches for new physics in top quark sector by Tevatron and mostly LHC Run I data are discussed.

\section{New Physics Searches in Top Quark Production}

Many theories beyond SM predict massive charged gauge bosons which may exist as partners to the SM gauge bosons. The $W^{\prime}$ boson is one such particle that can manifest itself at the proton proton collisions at LHC. Both CMS [2] and ATLAS [3] collaborations search for the evidence of the $W^{\prime} \rightarrow t b$ in both leptonic $[4,5]$ and hadronic $[6,7]$ final states. The invariant mass distribution for $W^{\prime}$ is reconstructed using the mass of top and bottom quarks, $M_{t b}$. In lepton+jets channel, the $W$ mass constraint is used to reconstruct top mass. Jet substructure algorithm is used by both experiments to analyze the boosted particle topology in hadronic final state. Assuming right handed coupling to quarks, a limit is set on the production cross section of $W^{\prime}$ comparing observed and predicted $M_{t b}$ distributions for different $W^{\prime}$ mass values.

The combined lower mass limit from leptonic and hadronic channels for right handed $W^{\prime}$ given by ATLAS is $1.92 \mathrm{TeV}$ with $95 \% \mathrm{CL}$ at centre of mass energy $\sqrt{s}=8 \mathrm{TeV}$. CMS excluded a right handed $W^{\prime}$ with mass below $2.0 \mathrm{TeV}$ in hadronic channel and $2.67 \mathrm{TeV}$ in leptons+jets channel with $95 \% \mathrm{CL}$ at $\sqrt{s}=13 \mathrm{TeV}$. Tevatron has also performed a similar search for massive $W^{\prime}$ particle at $\sqrt{s}=1.97 \mathrm{TeV}$, but in the smaller mass spectrum in leptonic final state. It excluded a right handed $W^{\prime}$ below $860 \mathrm{GeV}$ mass with 95\% CL [9]. ATLAS also excluded the existence of left handed $W^{\prime}$ boson with mass less than 1.8(1.7) TeV without(with) interference from SM $t W$ production.

The production of chromophilic heavy neutral vector boson $Z^{\prime}$ which decays to gluons is studied by CDF [8] collaboration at $\sqrt{s}=1.97 \mathrm{TeV}$ [10]. $Z^{\prime} \rightarrow g^{*} g \rightarrow t \bar{t} g$ mass is reconstructed in semi-leptonic final state. The production cross section is constrained within a range of 40-300 fb for $Z^{\prime}$ mass values $500-1000 \mathrm{GeV}$.
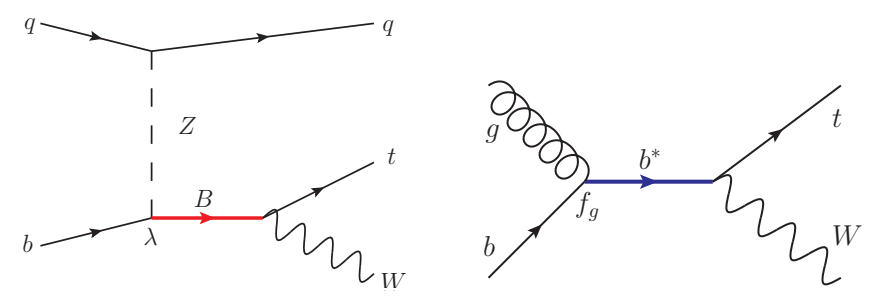

Figure 1: Leading order Feynman diagrams for the production and decay of $B$ and $b^{*}$

In beyond SM theories, vector-like quarks or VLQs are expected to preferentially couple to third generation quarks, namely, the top quark. ATLAS has searched for singly produced bottom 
type VLQs and excited quarks decaying through top $\left(B / b^{*} \rightarrow W t\right)$ at $\sqrt{s}=8 \mathrm{TeV}$ [14], assuming that $B$ is produced via the weak interaction whereas $b^{*}$ is produced via strong interaction, as shown in Fig. 1. In the analysis with leptonic final states, no constraint can be put on $B$ mass, but $b^{*}$ mass could be excluded below 1.5 TeV. Decays of pair produced VLQs are studied by both CMS and ATLAS experiments. An up-type quark $T$ can decay through a Higgs or a $Z$ boson and a top quark ( $T \rightarrow H t, Z t$ ), in addition to the expected $T \rightarrow W b$ channel. Similarly, a bottom type $B$ quark can decay though a Higgs or a $Z$ boson and $b$ quark $(B \rightarrow H b, Z b)$, as well as $B \rightarrow W t$. ATLAS performed a search for both $T$ and $B$ quark in lepton+jets final state at $\sqrt{s}=8 \mathrm{TeV}$ [11]. Observed 95\% CL lower limit on $T$ mass is $730-950 \mathrm{GeV}$ for all possible branching fractions in the three decay modes. Similarly for all possible decays the $95 \%$ lower limit on $B$ mass is $575-813 \mathrm{GeV}$. CMS observed a 95\% CL lower limit on $T$ mass at $860 \mathrm{GeV}$ in leptonic final states of pair produced $T$ quarks at $\sqrt{s}=13 \mathrm{TeV}$ [12]. Massive vector boson decay $Z^{\prime} \rightarrow T^{\prime} t \rightarrow W b W b$ is also studied by CMS at $\sqrt{s}=13 \mathrm{TeV}$ [13], in all hadronic final state. The $T^{\prime}$ and $Z^{\prime}$ invariant mass is reconstructed using jet substructure techniques. The production cross section for $Z^{\prime}$ is constrained below 0.13-11 pb with 95\% CL for different mass hypotheses.
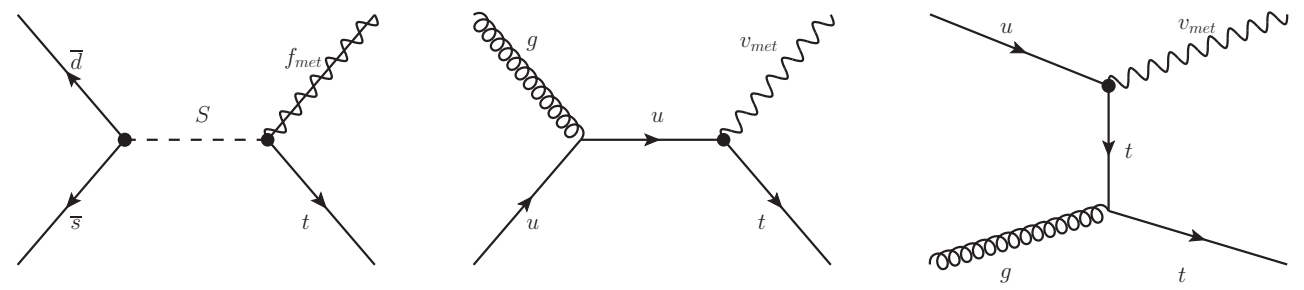

Figure 2: Feynman diagrams of leading order processes leading to mono-top events

Certain supersymmetric invisible particles can be produced together with a top quark as predicted by both resonant and non-resonant models, as shown in Fig. 2. In the resonant model, a heavy scalar particle $S$ can decay to a top and an invisible fermion $\left(S \rightarrow t f_{m e t}\right)$ which appears as missing transverse energy or MET. In the non-resonant model, an invisible vector particle $v_{\text {met }}$ is produced together with a top. The possibilities of the above processes is examined by the ATLAS experiment in a final state where the top decays leptonically and the event has significant amount of MET at $\sqrt{s}=8 \mathrm{TeV}$ [15]. 95\% CL upper limits are set on the production cross section and the corresponding coupling strength for various mass values of the invisible particle. For the production of $\mathrm{S}$ with mass $500 \mathrm{GeV}$, effective coupling strength $a_{\text {res }}$ is excluded above 0.15 for $f_{\text {met }}$ mass 0-100 GeV. In the non-resonant production, coupling strength $a_{n o n-r e s}$ is excluded above 0.2 for $v_{\text {met }}$ mass $0-657 \mathrm{GeV}$.

Dark matter has been searched in models where a scalar/psedoscalar particle is produced in association with a top quark pair, and it decays through invisible particles ( $p p \rightarrow t \bar{t} \phi, \phi \rightarrow$ MET). Leptonic final states with high MET is studied by CMS experiment at $\sqrt{s}=13 \mathrm{TeV}$ [16], which puts a lower limit on the scalar/pseudoscalar mass at $39 \mathrm{GeV}$, assuming the invisible particle mass to be $1 \mathrm{GeV}$. 


\section{New Physics Searches in Top Quark Decay}

If there exists a light charged Higgs, as suggested by beyond SM theories such as MSSM, it can be produced through top quark decays as shown in Fig. 3. CMS and ATLAS both performed a search for $t \rightarrow H^{+} b$ when $m_{H^{+}}<m_{t}$. ATLAS studied $H^{+} \rightarrow \tau v$ with three final states: all hadronic,

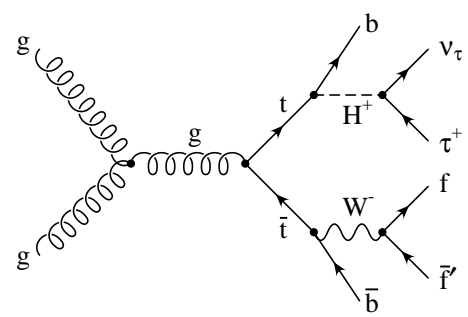

Figure 3: Feynman diagram for light charged Higgs production

semi-leptonic and fully leptonic depending on the decays of $W$ and $\tau$ at $\sqrt{s}=7 \mathrm{TeV}$ [17]. Assuming $\mathrm{BR}\left(H^{+} \rightarrow \tau v\right)=100 \%$ the $\mathrm{BR}\left(t \rightarrow H^{+} b\right)$ is constrained below $1 \%-5 \%$ for different Higgs mass. Charged Higgs can also decay through $c \bar{s}$ which is studied by CMS in lepton+jets final state at $\sqrt{s}=8 \mathrm{TeV}$ [18]. Assuming $\mathrm{BR}\left(H^{+} \rightarrow c \bar{s}\right)=100 \%$ the $\mathrm{BR}\left(t \rightarrow H^{+} b\right)$ is constrained below $1.2 \%$ $6.5 \%$ for different values of $m_{H^{+}}$.

\section{Searches for Anomalous Couplings of Top Quark}

If there exists anomalous coupling of Higgs with top, it can be studied through the single top production with Higgs. For example, the single top production with Higgs can hint at whether the relative sign between the fermion coupling and vector boson coupling are according to SM prediction. In case of inverted top coupling, the cross section will be enhanced. CMS studied this process in a direct search dedicated to the $t H q$ channel at $\sqrt{s}=8 \mathrm{TeV}$ [19], where the observed 95\% CL upper limit on cross section is 2.8 times of that predicted by SM. The anomalous coupling is studied through the $H \rightarrow \gamma \gamma$ decay by ATLAS using $\sqrt{s}=7$ and $8 \mathrm{TeV}$ data [20]. The analysis takes into account the contributions from both $t \bar{t} H$ and $t H$ processes. The $t t H$ coupling is constrained within the range -1.3 to 8.0 .
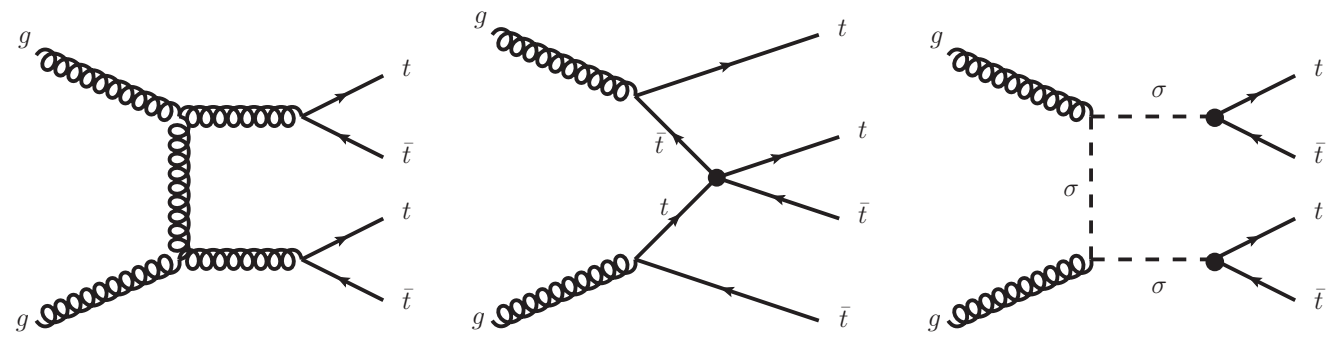

Figure 4: Feynman diagrams of four top production processes

The production of four tops is a rare process in $\mathrm{SM}\left(\sigma_{S M} \sim 1 \mathrm{fb}\right.$ at $\left.\sqrt{s}=8 \mathrm{TeV}\right)$; however, if there exists quartic top coupling or some scalar particles that decay through top quark pairs as 
shown in Fig. 4, the cross section may increase. Both CMS and ATLAS experiments studied this channel in the lepton+jets final state [21,22]. Oppsite sign di-lepton final states are also studied in the CMS analysis. The cross section limit given by ATLAS is 23 times $\sigma_{S M}$ at $\sqrt{s}=8 \mathrm{TeV}$ and the CMS limit is 10 times $\sigma_{S M}$ at $\sqrt{s}=13 \mathrm{TeV}$. At $\sqrt{s}=8 \mathrm{TeV}$ the CMS limit was 32 times the SM cross section [23].

The angular properties of the top decay products can be studied through the $W t b$ vertex structure. They can be used to constrain the polarization fractions, since top is the only quark that decays before hadronization. There are also flavour changing neutral current or FCNC interactions of top in both production and decay processes. These topics are discussed at length in other presentations of this conference $[24,25]$.

\section{Conclusions}

Tevatron and LHC Run I results from a variety of processes involving top quarks constrain a large number of beyond SM scenarios. Till now the studies have mostly been statistics limited and Standard Model holds strong. With the $\sim 100 \mathrm{fb}^{-1}$ of data that will be collected by the end of LHC Run II, it will bring in new possibilities for both SM and beyond.

\section{References}

[1] LHC/Tevatron NOTE [arXiv:1403.4427].

[2] CMS Collaboration,The CMS experiment at the CERN LHC, JINST3:S08004, 2008.

[3] ATLAS Collaboration, The ATLAS Experiment at the CERN Large Hadron Collider, JINST3:S08003, 2008.

[4] CMS Collaboration, CMS-PAS-B2G-16-017.

[5] ATLAS Collaboration, Phys. Lett. B 743 (2015) 235-255, [arXiv:1410.4103].

[6] CMS Collaboration, CMS-PAS-B2G-16-009.

[7] ATLAS Collaboration, EPJC (2015) 75:165, [arXiv:1408.0886].

[8] CDF Collaboration, The CDF Detector: An Overview, NIM A271 (1988) 387-403.

[9] CDF Collaboration, Phys. Rev. Lett. 115, 061801 (2015), [arXiv:1504.01536].

[10] CDF Collaboration, Phys. Rev. D 86, 112002 (2012).

[11] ATLAS Collaboration, JHEP 08 (2015) 105, [arXiv:1505.04306].

[12] CMS Collaboration, CMS-PAS-B2G-16-011.

[13] CMS Collaboration, CMS-PAS-B2G-16-013.

[14] ATLAS Collaboration, JHEP 02 (2016) 110, [arXiv:1510.02664].

[15] ATLAS Collaboration, Eur. Phys. J. C (2015) 75:79, [arXiv:1410.5404].

[16] CMS Collaboration, CMS-PAS-EXO-16-028.

[17] ATLAS Collaboration, JHEP 06 (2012) 039, [arXiv:1204.2760].

[18] CMS Collaboration, JHEP 12 (2015) 178, [arXiv:1510.04252]. 
[19] CMS Collaboration, JHEP 06 (2016) 177, [arXiv:1509.08159].

[20] ATLAS Collaboration, Phys. Lett. B 740 (2015) 222-242, [arXiv:1409.3122].

[21] CMS Collaboration, CMS-PAS-TOP-16-016.

[22] ATLAS Collaboration, JHEP 08 (2015) 105, [arXiv:1505.04306].

[23] CMS Collaboration, JHEP 11 (2014) 154, [arXiv:1409:7339].

[24] Proceedings of CKM 2016 on Single top quark and Vtb measurements, CMS CR-2017/025.

[25] Proceedings of CKM 2016 on Flavor Changing Neutral Current searches in the top quark sector, CMS CR-2017/097. 